\title{
A NuSTAR census of coronal parameters in Seyfert galaxies
}

\author{
A. Tortosa ${ }^{1}$, S. Bianchi ${ }^{1}$, A. Marinucci ${ }^{1}$, G. Matt $^{1}$, and P. O. Petrucci ${ }^{2}$ \\ ${ }^{1}$ Dipartimento di Matematica e Fisica, Universitá degli Studi Roma Tre, via della Vasca Navale 84, 00146 Roma, Italy \\ e-mail: tortosa@fis.uniroma3.it \\ ${ }^{2}$ Univ. Grenoble Alpes, CNRS, IPAG, 38000 Grenoble, France
}

Received 29 November 2017 / Accepted 12 January 2018

\begin{abstract}
Context. We discuss the results of the hot corona parameters of active galactic nuclei (AGN) that have been recently measured with NuSTAR. The values taken from the literature of a sample of 19 bright Seyfert galaxies are analysed.

Aims. The aim of this work is to look for correlations between coronal parameters, such as the photon index and cut-off energy (when a phenomenological model is adopted) or the optical depth and temperature (when a Comptonization model is used), and other parameters of the systems, such as the black hole mass or the Eddington ratio.

Methods. We analysed the coronal parameters of the 19 unobscured, bright Seyfert galaxies that are present in the Swift/BAT 70-month catalogue and that have been observed by NuSTAR, alone or simultaneously with others X-ray observatories, such as Swift, Suzaku, or XMM-Newton.

Results. We found an anti-correlation with a significance level $>98 \%$ between the coronal optical depth and the coronal temperature of our sample. On the other hand, no correlation between the above parameters and the black hole mass, the accretion rate, and the intrinsic spectral slope of the sources is found.
\end{abstract}

Key words. galaxies: active - galaxies: Seyfert - X-rays: galaxies - black hole physics

\section{Introduction}

The X-ray continuum of active galactic nuclei (AGN) can be explained by thermal Comptonization of the soft UV radiation, produced by the inner accretion disc, by a plasma of relativistic electrons around the supermassive black hole, known as the corona (Haardt \& Maraschi 1993). This continuum is reprocessed by cold neutral circumnuclear medium (e.g. the accretion disc or the molecular torus) and gives rise to a reflection bump at around $30 \mathrm{keV}$ and an iron $\mathrm{K} \alpha$ line emission at around $6.4 \mathrm{keV}$ (e.g. Matt et al. 1991). The presence of these features in the spectrum places constraints on the geometry of the $\mathrm{X}$-ray emitting region, and tells us that the hot plasma region is quite compact and likely situated close to the accretion disc.

However, the detailed geometry of the disc-corona system is still largely unknown, and indeed the size, location, and shape of the corona are still a matter of debate. It is not yet clear if the corona is very compact, as assumed in the lamp-post geometry (Matt et al. 1991; Henri \& Petrucci 1997; Petrucci \& Henri 1997; Miniutti \& Fabian 2004), or more extended, filling the inner part of the accretion flow. It is also not yet clear if the coronal plasma is a continuous or a patchy medium (Petrucci et al. 2013).

To solve the doubts raised above we need to study the broadband X-ray spectrum of AGN in detail, to disentangle all the complex spectral features in this energy range, to remove all the degeneracies between the primary continuum features and other physical observables in order to constrain the coronal parameters, and to have an overview of the physics and the structure of the hot corona (Marinucci et al. 2016).

The simplest way to obtain a description of Comptonizing coronae is to measure the cut-off in the hard X-ray spectrum and the photon power-law index. The spectral cut-off is directly related to the Comptonizing electron temperature of the corona, while the power-law index depends on the interplay between the electron temperature and the optical depth. These measurements have been performed with hard X-ray satellites, such as BeppoSAX (Dadina 2007; Perola et al. 2002), INTEGRAL (Panessa et al. 2011; de Rosa et al. 2012; Molina et al. 2013; Molina et al. 2009a), and Swift (Ricci et al. 2017). The cut-off values range between 50 and $300 \mathrm{keV}$. The high-energy cutoff values were found to correlate with the photon index of the primary emission. It is not clear, however, if this is a real correlation of an effect due to the degeneracies between parameters in those background-dominated observations.

The Nuclear Spectroscopic Telescope Array (NuSTAR, Harrison et al. 2013) is a hard X-ray observatory that was launched in June 2012. It has two coaligned X-ray optics which focus X-ray photons onto two independent shielded focal plane modules, namely FPMA and FPMB. Thanks to its focusing optics, it has a broad and high-quality spectral coverage from 3 to $79 \mathrm{keV}$. Given these features, NuSTAR is suitable for studying the hard X-ray spectra of AGN with high sensitivity, discriminating between the primary X-ray emission and the scattered or reflected component (i.e. radiation which interacts with circumnuclear gas and gets absorbed or Compton scattered). Alone or with simultaneous observations with other X-ray observatories operating below $10 \mathrm{keV}$, such as XMM-Newton, Suzaku, and Swift, it has provided strong constraints on the coronal properties of many AGN (Brenneman et al. 2014; Baloković et al. 2015; Fabian et al. 2015; Matt et al. 2015; Marinucci et al. 2014a, 2016; Tortosa et al. 2017; Fabian et al. 2017).

To better understand the complex environment of AGN, it is important to search for correlations between coronal parameters and other physical parameters, such as the black hole mass and the Eddington ratio. In this paper, we present the analysis of a small catalogue of AGN, built up by choosing the 
unobscured nearby, non-jetted Seyfert galaxies (following the distinction made by Padovani et al. 2017) that have been observed by NUSTAR (often in coordination with XMM-Newton, Suzaku, or Swift). We took the values of the coronal parameters of this sample of AGN from the literature. The aim of the paper is to list and discuss these values and look for possible correlations.

\section{The sample}

The primary X-ray emission is characterized by a power-law spectral shape extending to energies determined by the electron temperature. The power law often shows a cut-off at high energies. Both the energy of the cut-off and the photon index are related to the temperature and the optical depth of the corona. Comptonization models imply that the cut-off energies are 2 3 times the temperature of the corona (Petrucci et al. 2000, 2001). To investigate the shape of the spectrum it is important to take into account the reprocessed emission of the circumnuclear environment in this energy range, such as reflection from the accretion disc and distant material. Typical X-ray features of the cold circumnuclear material include the intense iron $\mathrm{K} \alpha$ line at $6.4 \mathrm{keV}$ and the associated Compton reflection peaking at $30 \mathrm{keV}$.

Unlike the previous hard X-ray observatories, which are background dominated for almost all AGN, NuSTAR is the first focusing hard X-ray telescope on orbit, 100 times more sensitive in the 10-79 $\mathrm{keV}$ band than the previous observatories working in the same energy band. The focusing capability implies a very low background, and the observation of bright AGN are source-dominated. NUSTAR data can, therefore, provide strong and robust constraints on the high-energy cut-off, allowing us to study AGN at high energies with high precision and with unprecedented accuracy. Thanks to NUSTAR observations in collaboration with other X-ray satellites, such as XMM-Newton and Swift, in the last few years several cut-off energies have been measured with very high precision.

We built the catalogue by choosing the unobscured $\left(N_{H} \leq 6 \times 10^{22} \mathrm{~cm}^{-2}\right)$ nearby brightest Seyfert galaxies that are present in the Swift/BAT 70-month catalogue and that have been observed by NUSTAR alone or simultaneously with other X-rays observatories, such as Swift, Suzaku, or XMM-Newton. We selected only unobscured or moderately obscured AGN to have a clear view of the primary emission component. Other objects for which the cut-off energy had been left fixed in the spectral analysis are not included (e.g. 1H0707-495), since they need a more intensive study of this issue. The list and the characteristics of all the sources can be found in Table 2 and in Appendix B.

The final sample comprises 19 objects, 12 with a measurement of the cut-off energy and 7 having only a lower limit. The distribution of high-energy cut-off measurements from the sample is shown in Fig. 1.

\section{Black hole mass measurements}

Some of the selected sources had more than one literature value for the mass of the central black hole. One of the most reliable and direct ways to measure the mass of a supermassive black hole residing in the nucleus of an active galaxy is reverberation mapping (RM, Blandford \& McKee 1982; Peterson 1993). We decided to use the RM mass values for the sources that have one (IC4329A, 3C390.3, Ark 564, Ark 120, Mrk 335, Fairall 9, Mrk 766, PG 1211+143, Peterson et al. 2004; NGC 6814, Pancoast

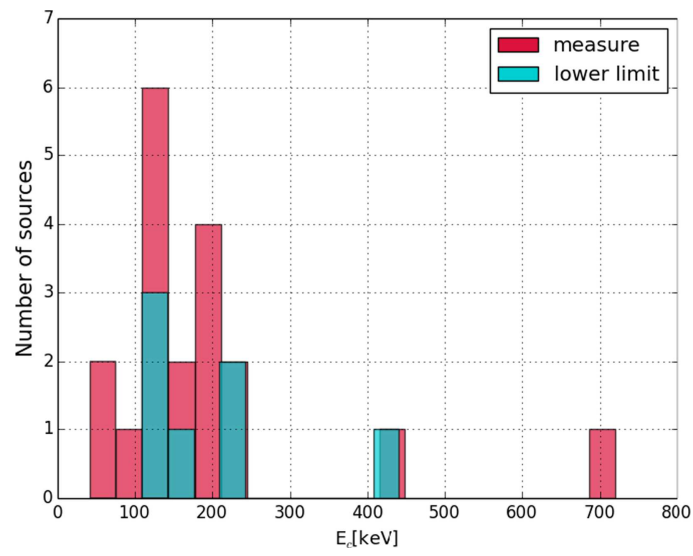

Fig. 1. Distribution of the high-energy cut-off of the sample when both measures (red) and lower limits (blue) are considered.

Table 1. Correlations factor, $\rho$, and null hypothesis probability, $h_{0}$.

\begin{tabular}{ccccc}
\hline \hline$X$ & $Y$ & $\rho$ & $h_{0}$ & Geometry \\
\hline$\Gamma$ & $E_{c}$ & 0.18 & 0.47 & - \\
$\log \left(M_{\mathrm{bh}} / M_{\odot}\right)$ & $E_{c}$ & -0.11 & 0.61 & - \\
$L_{\mathrm{bol}} / L_{\mathrm{Edd}}$ & $E_{c}$ & -0.14 & 0.56 & - \\
$\tau$ & $k T_{e}$ & -0.88 & 0.004 & Slab \\
$\tau$ & $k T_{e}$ & -0.63 & 0.02 & Sphere \\
$\log \left(M_{\mathrm{bh}} / M_{\odot}\right)$ & $\tau$ & -0.22 & 0.63 & Slab \\
$\log \left(M_{\mathrm{bh}} / M_{\odot}\right)$ & $\tau$ & -0.26 & 0.46 & Sphere \\
$L_{\mathrm{bol}} / L_{\mathrm{Edd}}$ & $\tau$ & 0.49 & 0.27 & Slab \\
$L_{\mathrm{bol}} / L_{\mathrm{Edd}}$ & $\tau$ & 0.38 & 0.28 & Sphere \\
$\log \left(M_{\mathrm{bh}} / M_{\odot}\right)$ & $k T_{e}$ & 0.20 & 0.64 & Slab \\
$\log \left(M_{\mathrm{bh}} / M_{\odot}\right)$ & $k T_{e}$ & 0.18 & 0.47 & Sphere \\
$L_{\mathrm{bol}} / L_{\mathrm{Edd}}$ & $k T_{e}$ & -0.37 & 0.41 & Slab \\
$L_{\mathrm{bol}} / L_{\mathrm{Edd}}$ & $k T_{e}$ & -0.36 & 0.32 & Sphere \\
\hline
\end{tabular}

et al. 2014, 2015). For the sources without a RM measurement we used mass values derived from virial mass methods, such as the single-epoch (SE) method. These methods start from the relation between the size and the luminosity of the broad-line region ( $R-L$ relation) to derive the broad-line region size through a single measure of the optical continuum luminosity; combining this information with the width of a broad line, it is possible to build a relation with the black hole mass (Vestergaard 2002; Vestergaard \& Peterson 2006).

One of the most used $R-L$ relations based on $\mathrm{H} \beta \mathrm{RM}$ measurements is (Bentz et al. 2009)

$\log \frac{R}{\text { lightdays }}=-2.13+0.519 \log \frac{\lambda L_{\lambda}(5100 \AA)}{\operatorname{erg~s}^{-1}}$.

In the case of NGC 5506 the central stellar velocity dispersion $\left(\approx 180 \mathrm{~km} \mathrm{~s}^{-1}\right)$ (Oliva et al. 1999; Papadakis 2004) and the width of the [OIII] line (Boroson 2003) give a black hole mass of $\sim 10^{8} M_{\odot}$, and we decided to use this value.

We assumed a $20 \%$ uncertainty for black hole mass estimates not inferred from reverberation.

\section{Fitting process}

The aim of this work is, as said before, to look for correlations between the spectral parameters, such as the cut-off value and physical parameters. 
A. Tortosa et al.: A NUSTAR census of coronal parameters in Seyfert galaxies

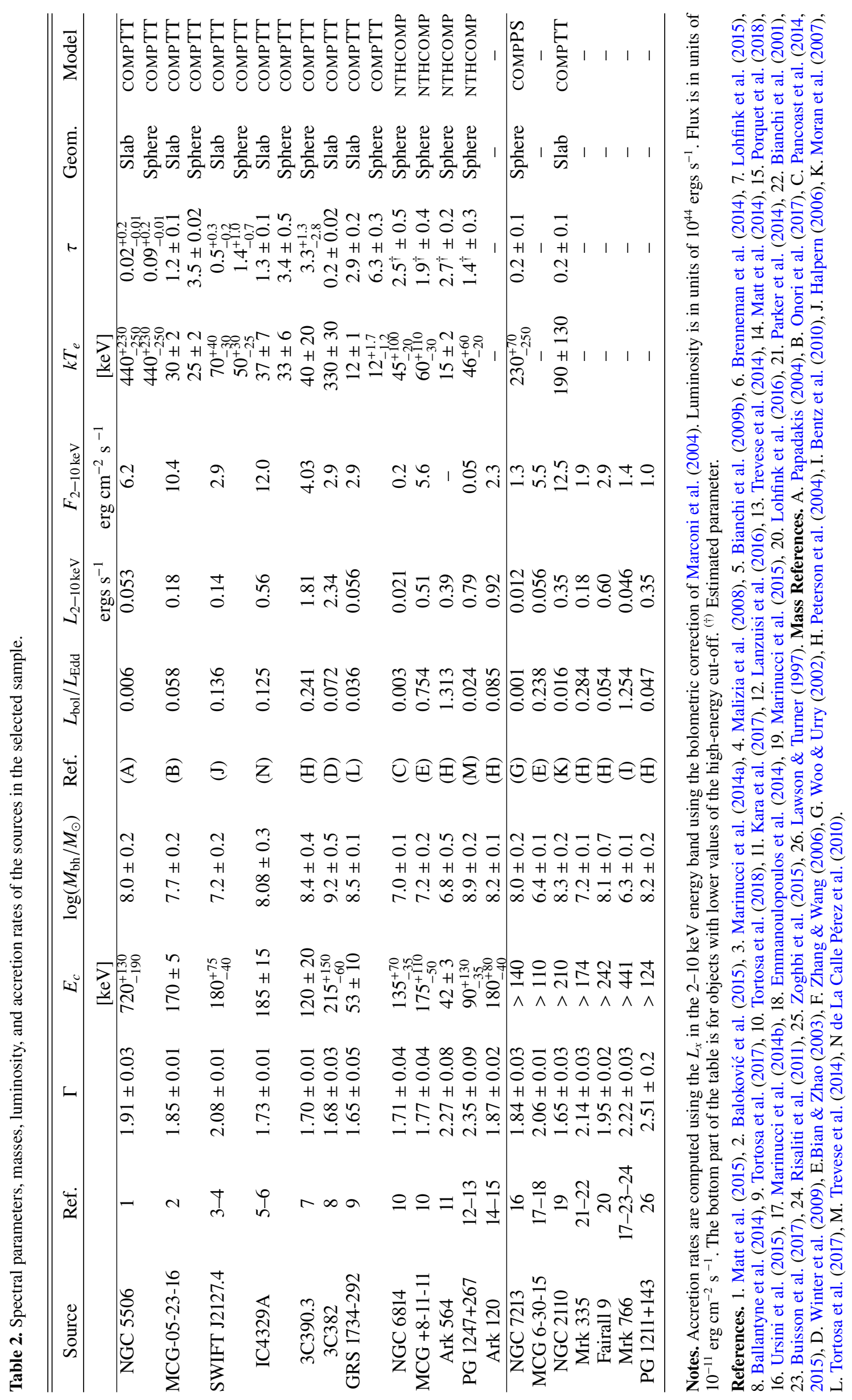

A37, page 3 of 9 


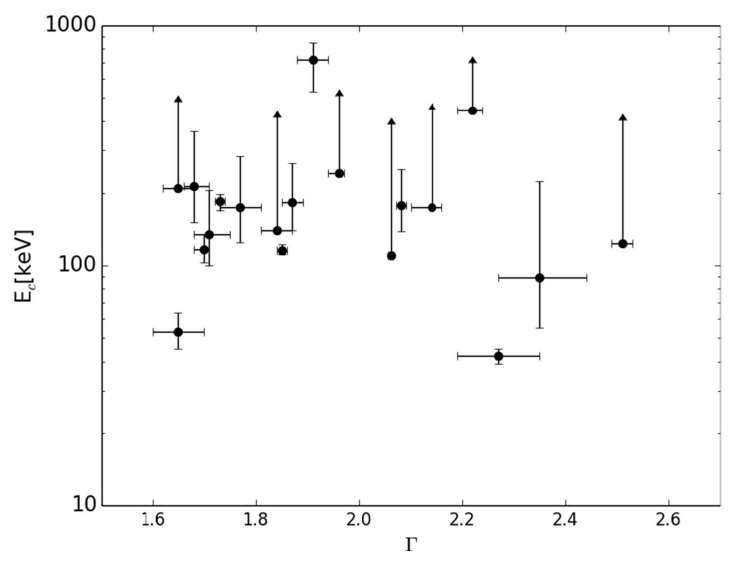

Fig. 2. High-energy cut-off vs the photon index of the sample.

The goodness of the correlation is given by Spearman's rank correlation coefficient or Spearman's $\rho$. The Spearman correlation coefficient is defined as the Pearson correlation coefficient between the ranked variables. The sign of the Spearman correlation indicates the direction of the association between $X$ (the independent variable) and $Y$ (the dependent variable). A Spearman correlation close to zero indicates that there is no tendency for $Y$ to either increase or decrease when $X$ increases. When $X$ and $Y$ are perfectly monotonically related, the Spearman correlation coefficient becomes 1.0 (or -1.0 for anti-correlation).

In the fitting process the "null hypothesis" is also given. The null hypothesis, denoted by $h_{0}$, is the probability that sample observations result purely from chance. A small $h_{0}$ value indicates a significant correlation. The fits are made using the Interactive Data Language (IDL ${ }^{1}$ ) programming language. We fitted the parameters with a simple linear relation in logarithmic scale:

$\log (y)=\mathbf{a} \log (x)+\mathbf{b}$

The fits are made with a Monte Carlo method which repeated the fit procedure by random sampling the values between a minimum value and a maximum value, which are identified respectively with the lower and the upper extreme of the errors of the measure (Bianchi et al. 2009a).

\subsection{Spectral parameters}

We started by looking for a correlation between the photon index $\Gamma$ and the high-energy cut-off with the relation of Eq. (2). As can be seen in Fig. 2, no statistically significant correlation is found between this parameters, in contrast with what was found by previous satellites (e.g. Perola et al. 2002; Ricci et al. 2017). This, together with the absence of a correlation between the high energy cutoff and the black hole mass and Eddington ratio of the sources in our sample (see Figs. 3 and 4), is reassuring, suggesting that with $N U S T A R$, and using a relatively large sample of well-exposed sources with good measurements, the intrinsic degeneracy between these two parameters is significantly reduced.

The following step was to search for a linear correlation between the high-energy cut-off and either the mass of the central black hole or the Eddington ratio.

$\overline{1}$ www.harrisgeospatial . com/SoftwareTechnology/IDL.aspx

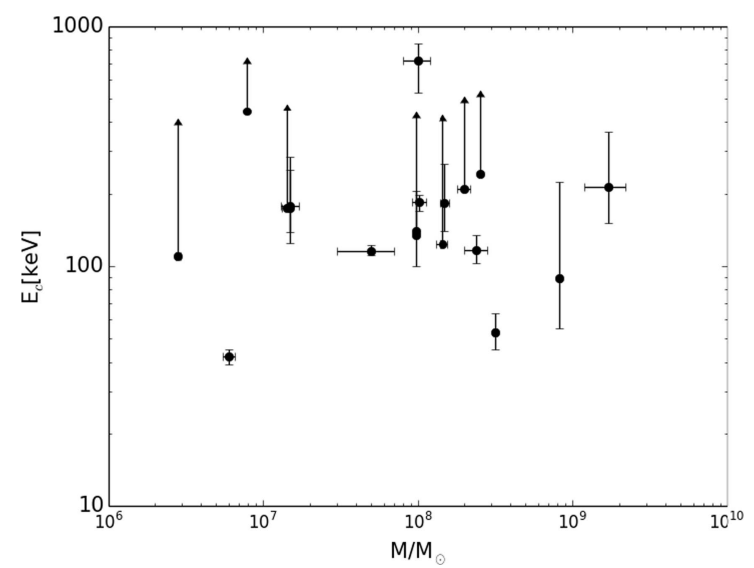

Fig. 3. High-energy cut-off vs the black hole mass of the sample.

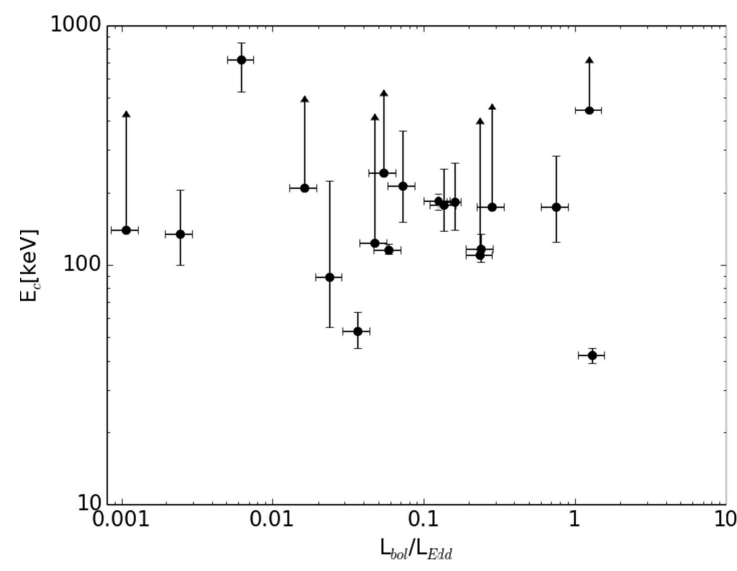

Fig. 4. High-energy cut-off vs Eddington ratio of the sample.

The Eddington ratio $L_{\mathrm{bol}} / L_{\mathrm{Edd}}$ is computed using the 2-10 keV absorption-corrected luminosity of the source to estimate the bolometric luminosity using the $2-10 \mathrm{keV}$ bolometric correction of Marconi et al. (2004). Error bars on the Eddington ratio are derived from uncertainties on the black hole mass and $2-10 \mathrm{keV}$ luminosity measurements. All values are listed in Table 2. The Spearman's $\rho$ values (Table 1) show that there is no significant correlation between the checked parameters.

\subsection{Physical parameters}

We consider the physical parameters that characterize the AGN coronae: the coronal temperature $k T_{e}$ and the optical depth. The distribution of these two values in our sample is shown in Fig. 5. It should be noted that for some of the sources the optical depth parameter is not directly measured since the model used (NTHCOMP inXSPEC) does not have the optical depth as a free parameter. In these cases, the optical depth has been estimated using the relation from Beloborodov (1999),

$\Gamma \approx \frac{9}{4} y^{-2 / 9}$

where $\Gamma$ is the photon index of the spectrum between 2 and $10 \mathrm{keV}$. The dependence on the optical depth is in the relativistic $y$-parameter,

$y=4\left(\Theta_{\mathrm{e}}+4 \Theta_{\mathrm{e}}^{2}\right) \tau(\tau+1)$ 

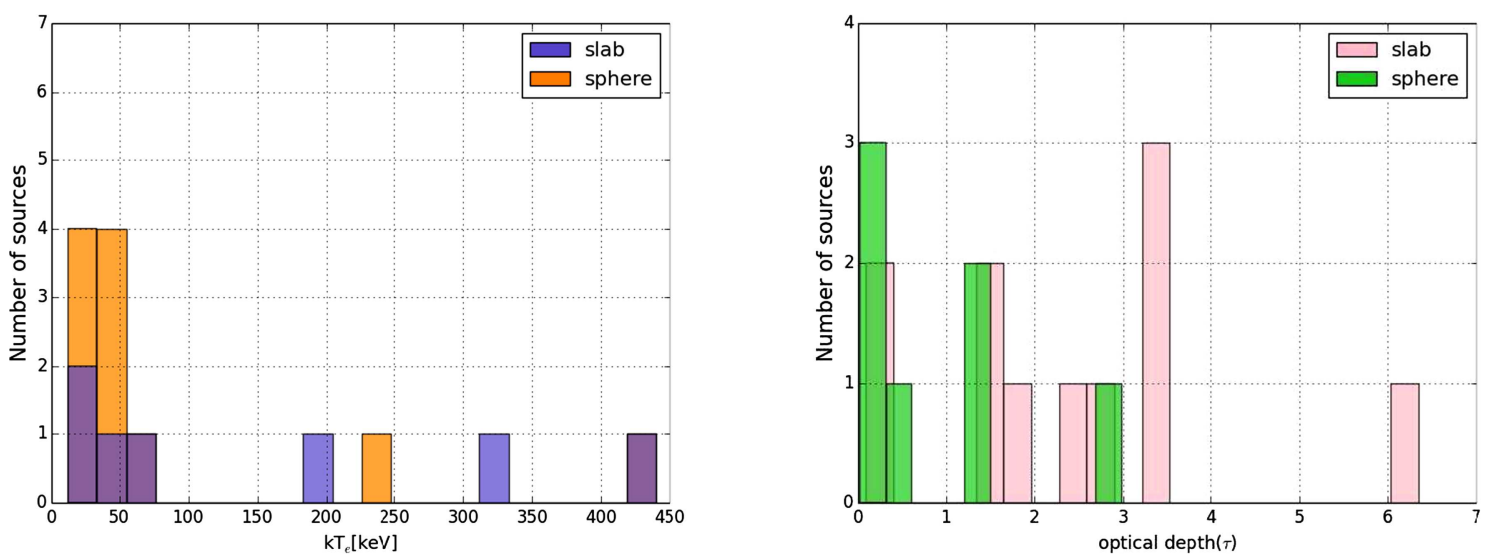

Fig. 5. Left panel: distribution of the coronal temperature values for the sources of the sample that have coronal temperature measurements. Right panel: distribution of the optical depth values for the sources in the sample that have direct or extrapolated measurements of this parameter. Both slab and sphere geometry of the corona are considered.

where $\Theta_{e}$ is the electron temperature normalized to the electron rest energy:

$\Theta_{e}=\frac{k T_{e}}{m_{e} c^{2}}$.

We performed the fit for the two cases of slab and spherical geometry of the corona.

In Appendix A, the results obtained with NTHCOMP are compared with those obtained with COMPTT for a few selected sources.

\subsection{Optical depth versus coronal temperature}

The optical depth and coronal temperature appear to be extremely anti-correlated. The Spearman correlation factor for this fit is $\rho=-0.88(-0.63)$ and the null hypothesis probability $h_{0}=0.004(0.03)$ for the slab (spherical) geometry; see also Fig. 6.

Using Eq. (2) we found, in the case of a slab geometry, the following intercept and slope values for the fit:

$\mathbf{a}=-0.7 \pm 0.1 ; \quad \mathbf{b}=1.6 \pm 0.06$

The parameters of the linear regression in the case of the spherical geometry are

$\mathbf{a}=-0.7 \pm 0.2 ; \quad \mathbf{b}=1.8 \pm 0.1$

This is a very interesting result, but the physical interpretation is not straightforward. We discuss this correlation in the following section.

We also searched for correlations between the above parameters (coronal optical depth and coronal temperature) and the central black hole of the AGN, and the Eddington ratio in slab and in spherical coronal geometries. We do not find statistically significant correlation in any of the analysed cases (see Table 1).

\section{Discussion}

We found two relevant results from this analysis. The first is the lack of correlation between the high-energy cut-off and the spectral photon index of the primary power law (see Fig. 2). Perola et al. in 2002 found a correlation between the high-energy
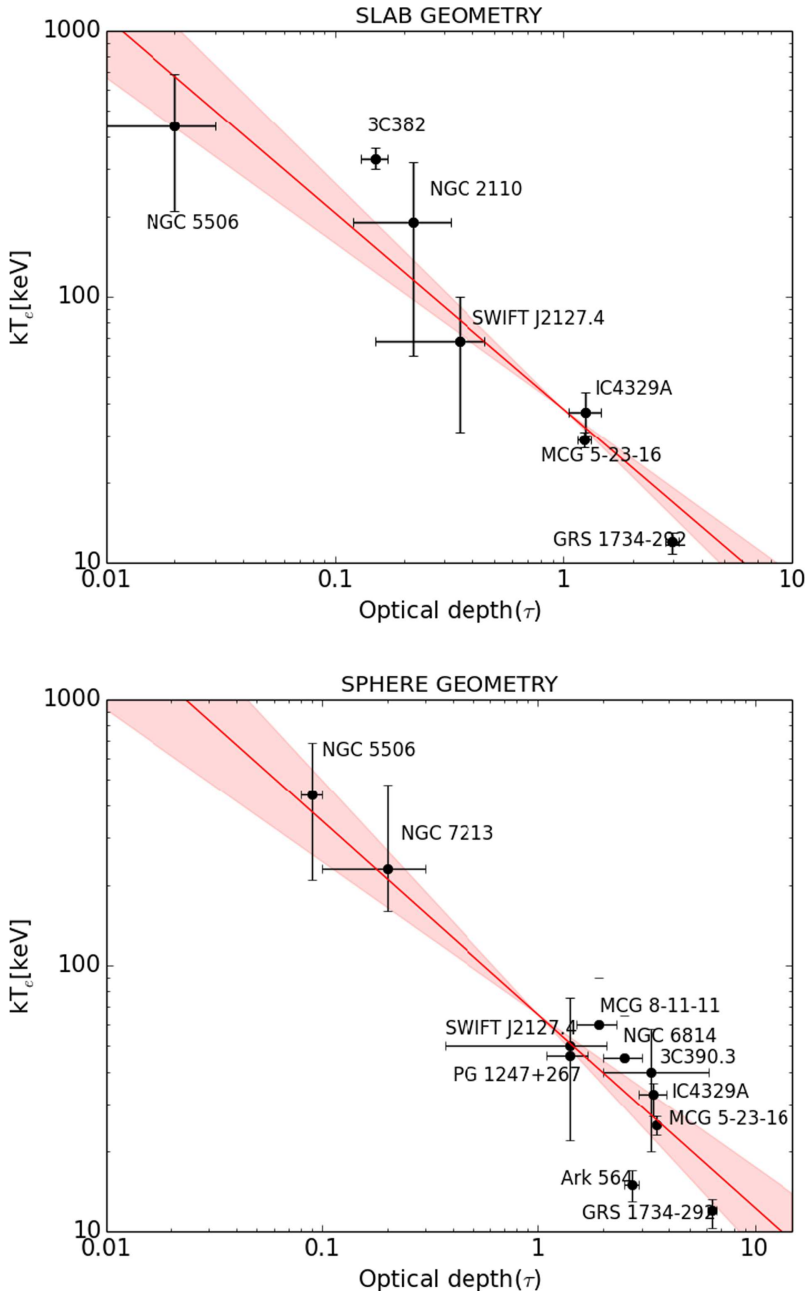

Fig. 6. Fit (red line) and error on the fitting relation (red shaded region) of the optical depth vs the electron coronal temperature in the case of a disc-shaped corona (top panel) and spherical corona (bottom panel). The fit is made using Eq. (2).

cut-off and the photon index of the primary power law with a correlation coefficient equal to 0.88 , with $E_{c}$ increasing with $\Gamma$. The same correlation is found in the Swift/BAT sample, where Ricci et al. (2017) found that, when fitting the simulated Swift/BAT 
spectra with a simple power-law model, the Swift/BAT photon index increases when the cut-off decreases (see Fig. 19 in their work).

Given that the two parameters are correlated in the fit procedure, this correlation may be an artefact due to a systematic error on one of the two parameters. Instead, we found no significant correlation between $\Gamma$ and $E_{c}$. The lack of correlation between these parameters confirms what found by Molina et al. (2009a) in the INTEGRAL complete sample of type 1 AGN. This result means that there are no large systematics in the NuSTAR measurements.

The second important result is the presence of a strong anticorrelation between the optical depth and the coronal temperature, both in the slab and in the spherical geometry. The interpretation of this anti-correlation is not trivial. Of course the values of the parameters are model dependent. We checked the $k T_{e}$ and $\tau$ values in the spherical geometry of the corona for some of the sources of the sample, in particular GRS 1734-292, NGC 5506, and MCG-05-23-16 which have very different values of temperature and optical depth, by reanalysing the NuSTAR observations. The coronal temperature and the optical depth of the three sources listed above were all obtained with the COMPTT model (Titarchuk 1994). Instead we used the NTHCOMP model (Zdziarski et al. 1996; Życki et al. 1999), see Appendix A. We found different values for the two parameters of the three sources, but they still follow the anti-correlation (see Fig. 7).

Moreover, we note that the model used for the analysis of the different sources in the literature is not always the same. This excludes the fact that the correlation could be an artefact due to the use of the same model for the analysis.

The $\tau-k T_{e}$ anti-correlation cannot be easily reconciled with a fixed disc-corona configuration in radiative balance. Indeed, such a configuration corresponds to a fixed cooling/heating ratio for the corona. In this case the corona temperature and optical depth have only to adjust themselves in order to ensure the constancy of this ratio, but there is no reason for $k T_{e}$ and/or $\tau$ to change. In other words, if the disc-corona configuration of all the Seyfert galaxies is the same and is also in radiative balance, we would expect $k T_{e}$ and/or $\tau$ to cluster around the same values for all the objects in our sample.

The observed correlation indicates that one (or both) of these hypotheses is wrong. The invalidation of the former (same disc-corona configuration) implies a geometrical variation of the accretion flow. The correlation could arise from the variations in the transition radius $\mathrm{R}_{t r}$ separating the inner corona and the outer disc or from the variation in the height $H$ of the corona above the disc, as in the lamppost configuration. A lower $R_{t r} / H$ would imply greater cooling from the disc and then a lower temperature (assuming the heating is the same). In this case the observed anti-correlation would indicate that objects like NGC 5506 have a higher $R_{t r} / H$ than objects like GRS 1734-292.

The invalidation of the radiative balance hypothesis could instead be the result of a variation in the fraction of thermal emission due to viscous dissipation (hereafter intrinsic emission) with respect to the total disc emission, which also includes reprocessing of the coronal radiation. Indeed, for a fixed disc-corona geometry, the radiative balance will change if this proportion varies. If it increases, the cooling of the corona will increase and the temperature will decrease. In this case the observed anti-correlation would indicate that the disc intrinsic emission in objects like NGC 5506 contribute less to the total disc emission than do objects like GRS 1734-292.

We note that for a pair-dominated corona, the opposite behaviour is expected since an increase in the cooling (which

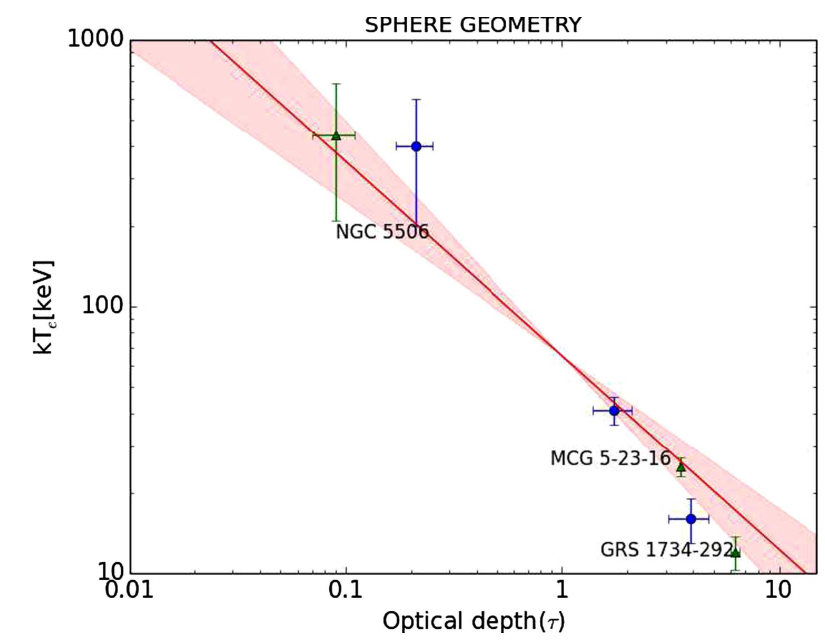

Fig. 7. Optical depth vs electron coronal temperature in the case of a spherical corona for GRS 1734-292, NGC 5506, and MCG-05-23-16 (blue circles) and the values obtained with our reanalysis (green triangle); see Appendix A. We superimposed the fit (red line) and the error on the fitting relation (red shaded region) obtained with all the literature values for all the sources, as in the lower panel of Fig. 6 .

is inversely proportional to the coronal optical depth, Haardt \& Maraschi 1991) would correspond to an increase in the corona temperature and not to a decrease (Ghisellini \& Haardt 1994). As a consequence, to explain the observed $k T_{e}-\tau$ anti-correlation, objects with high corona temperatures would have a smaller $R_{t r} / H$ or a larger contribution of the disc intrinsic emission than objects with low temperature.

\section{Conclusions}

We have presented and discussed recent high-energy cut-off measurements in a sample of 19 bright Seyfert galaxies observed by NUSTAR in collaboration with other X-ray observatories operating below $10 \mathrm{keV}$, such as XMM-Newton, Suzaku, and Swift. The goal of the work was to look for correlation between spectral and physical parameters in order to better understand the physics and the structure of AGN coronae.

This kind of analysis had already been done before the coming of NUSTAR using cut-off energy measurements performed by hard X-ray satellites like BeppoSAX (Perola et al. 2002) and INTEGRAL (Malizia et al. 2014). Unlike NuSTAR, these instruments are non-focusing, and are therefore background dominated for AGN observations.

We searched for correlations between the high-energy cutoff and the photon index of the primary power law, the mass of the central black hole, and the Eddington ratio $\left(L_{\text {bol }} / L_{\text {Edd }}\right)$. We did not find any statistically significant correlation between these parameters.

Finally, we searched for correlations between the physical parameters which characterize the hot coronae of AGN, i.e. the temperature and the optical depth, and the mass of the central black hole and the Eddington ratio. No significant statistical correlation is found between these parameters. Instead, a significant anti-correlation is found between the optical depth and the coronal temperature fit. We find a Spearman correlation coefficient $\rho=-0.88$ in the case of a slab geometry of the corona and -0.63 in the case of a spherical corona. The null hypothesis probability, $\rho$, is equal to 0.004 in the case of slab geometry and 0.02 for the sphere geometry. 
The observed anti-correlation suggests a disc-corona configuration in radiative balance, but requires differences, from source to source, in either the disc-corona configuration or in the intrinsic disc emission.

Acknowledgements. We thank the anonymous referee for the comments that helped to improve the clarity of the paper. This work made use of data from the NUSTAR mission, a project led by the California Institute of Technology, managed by the Jet Propulsion Laboratory, and funded by the National Aeronautics and Space Administration. We thank the NUSTAR Operations, Software, and Calibration teams for support with the execution and analysis of these observations. This research has made use of the NUSTAR Data Analysis Software (NuSTARDAS) jointly developed by the ASI Science Data Center (ASDC, Italy) and the California Institute of Technology (USA). AT, AM, and GM acknowledge financial support from the Italian Space Agency under grant ASI/INAF I/037/12/0-011/13, and SB under grant ASI-INAF I/037/12/P1. Part of this work is based on archival data, software, or online services provided by the ASI SCIENCE DATA CENTER (ASDC). AT, SB, AM, and GM acknowledge financial support from the European Union Seventh Framework Programme (FP7/20072013) under grant agreement no. 312789. POP acknowledges financial support from the CNES and the CNRS/PNHE. EP and SB acknowledge a financial contribution from the agreement ASI-INAF I/037/12/0.

\section{References}

Ballantyne, D. R., Bollenbacher, J. M., Brenneman, L. W., et al. 2014, ApJ, 794, 62

Baloković, M., Matt, G., Harrison, F. A., et al. 2015, ApJ, 800, 62

Beloborodov, A. M. 1999, in High Energy Processes in Accreting Black Holes, eds. J. Poutanen, \& R. Svensson, ASP Conf. Ser., 161, 295

Bentz, M. C., Peterson, B. M., Pogge, R. W., \& Vestergaard, M. 2009, ApJ, 694 L166

Bentz, M. C., Walsh, J. L., Barth, A. J., et al. 2010, ApJ, 716, 993

Bian, W., \& Zhao, Y. 2003, MNRAS, 343, 164

Bianchi, S., Matt, G., Haardt, F., et al. 2001, A\&A, 376, 77

Bianchi, S., Bonilla, N. F., Guainazzi, M., Matt, G., \& Ponti, G. 2009a, A\&A, 501, 915

Bianchi, S., Guainazzi, M., Matt, G., Fonseca Bonilla, N., \& Ponti, G. 2009b, A\&A, 495, 421

Blandford, R. D., \& McKee, C. F. 1982, ApJ, 255, 419

Boroson, T. A. 2003, ApJ, 585, 647

Brenneman, L. W., Madejski, G., Fuerst, F., et al. 2014, ApJ, 781, 83

Buisson, D. J. K., Lohfink, A. M., Alston, W. N., \& Fabian, A. C. 2017, MNRAS, 464, 3194

Dadina, M. 2007, A\&A, 461, 1209

Dauser, T., García, J., Parker, M. L., Fabian, A. C., \& Wilms, J. 2014, MNRAS, 444, L100

de La Calle Pérez, I., Longinotti, A. L., Guainazzi, M., et al. 2010, A\&A, 524, A50

de Rosa, A., Panessa, F., Bassani, L., et al. 2012, MNRAS, 420, 2087

Emmanoulopoulos, D., Papadakis, I. E., Dovčiak, M., \& McHardy, I. M. 2014, MNRAS, 439, 3931

Fabian, A. C., Vaughan, S., Nandra, K., et al. 2002, MNRAS, 335, L1

Fabian, A. C., Lohfink, A., Kara, E., et al. 2015, MNRAS, 451, 4375

Fabian, A. C., Lohfink, A., Belmont, R., Malzac, J., \& Coppi, P. 2017, MNRAS, 467, 2566

Filippenko, A. V., \& Halpern, J. P. 1984, ApJ, 285, 458

García, J., \& Kallman, T. R. 2010, ApJ, 718, 695

García, J., Dauser, T., Lohfink, A., et al. 2014, ApJ, 782, 76

Ghisellini, G., \& Haardt, F. 1994, ApJ, 429, L53

Gliozzi, M., Papadakis, I. E., Eracleous, M., et al. 2009, ApJ, 703, 1021

Grier, C. J., Peterson, B. M., Pogge, R. W., et al. 2012, ApJ, 744, L4

Haardt, F., \& Maraschi, L. 1991, ApJ, 380, L51

Haardt, F., \& Maraschi, L. 1993, ApJ, 413, 507

Halpern, J. P. 2006, ATel, 847

Harrison, F. A., Craig, W. W., Christensen, F. E., et al. 2013, ApJ, 770, 103

Henri, G., \& Petrucci, P. O. 1997, A\&A, 326, 87
Kalberla, P. M. W., Burton, W. B., Hartmann, D., et al. 2005, A\&A, 440, 775

Kara, E., García, J. A., Lohfink, A., et al. 2017, MNRAS, 468, 3489

Lanzuisi, G., Perna, M., Comastri, A., et al. 2016, A\&A, 590, A77

Lawson, A. J., \& Turner, M. J. L. 1997, MNRAS, 288, 920

Lohfink, A. M., Ogle, P., Tombesi, F., et al. 2015, ApJ, 814, 24

Lohfink, A. M., Reynolds, C. S., Pinto, C., et al. 2016, ApJ, 821, 11

Malizia, A., Bassani, L., Bird, A. J., et al. 2008, MNRAS, 389, 1360

Malizia, A., Molina, M., Bassani, L., et al. 2014, ApJ, 782, L25

Marconi, A., Risaliti, G., Gilli, R., et al. 2004, MNRAS, 351, 169

Marinucci, A., Matt, G., Kara, E., et al. 2014a, MNRAS, 440, 2347

Marinucci, A., Matt, G., Miniutti, G., et al. 2014b, ApJ, 787, 83

Marinucci, A., Matt, G., Bianchi, S., et al. 2015, MNRAS, 447, 160

Marinucci, A., Tortosa, A., \& NuSTAR AGN Physics Working Group. 2016, Astron. Nachr., 337, 490

Matt, G., Perola, G. C., \& Piro, L. 1991, A\&A, 247, 25

Matt, G., Marinucci, A., Guainazzi, M., et al. 2014, MNRAS, 439, 3016

Matt, G., Baloković, M., Marinucci, A., et al. 2015, MNRAS, 447, 3029

Miniutti, G., \& Fabian, A. C. 2004, MNRAS, 349, 1435

Molina, M., Bassani, L., Malizia, A., et al. 2009a, MNRAS, 399, 1293

Molina, M., Bassani, L., Malizia, A., et al. 2009b, MNRAS, 399, 1293

Molina, M., Bassani, L., Malizia, A., et al. 2013, MNRAS, 433, 1687

Moran, E. C., Barth, A. J., Eracleous, M., \& Kay, L. E. 2007, ApJ, 668, L31

Mukai, K., Hellier, C., Madejski, G., Patterson, J., \& Skillman, D. R. 2003, ApJ, 597, 479

Nagar, N. M., Oliva, E., Marconi, A., \& Maiolino, R. 2002, A\&A, 391, L21

Oliva, E., Origlia, L., Maiolino, R., \& Moorwood, A. F. M. 1999, A\&A, 350, 9

Onori, F. Ricci, F., La Franca, F., et al. 2017, MNRAS, 468, L97

Padovani, P., Alexander, D. M., Assef, R. J., et al. 2017, A\&ARv, 25, 2

Pancoast, A., Brewer, B. J., Treu, T., et al. 2014, MNRAS, 445, 3073

Pancoast, A., Brewer, B. J., Treu, T., et al. 2015, MNRAS, 448, 3070

Panessa, F., de Rosa, A., Bassani, L., et al. 2011, MNRAS, 417, 2426

Papadakis, I. E. 2004, MNRAS, 348, 207

Parker, M. L., Wilkins, D. R., Fabian, A. C., et al. 2014, MNRAS, 443, 1723

Pavlinsky, M. N., Grebenev, S. A., \& Sunyaev, R. A. 1992, Sov. Astron. Lett., 18,88

Perola, G. C., Matt, G., Cappi, M., et al. 2002, A\&A, 389, 802

Peterson, B. M. 1993, PASP, 105, 247

Peterson, B. M., Ferrarese, L., Gilbert, K. M., et al. 2004, ApJ, 613, 682

Petrucci, P. O., \& Henri, G. 1997, A\&A, 326, 99

Petrucci, P. O., Haardt, F., Maraschi, L., et al. 2000, ApJ, 540, 13

Petrucci, P. O., Haardt, F., Maraschi, L., et al. 2001, ApJ, 556, 716

Petrucci, P.-O., Paltani, S., Malzac, J., et al. 2013, A\&A, 549, A73

Porquet, D., Reeves, J. N., Matt, G., et al. 2018, A\&A, 609, A42

Poutanen, J., \& Svensson, R. 1996, ApJ, 470, 249

Ricci, C., Trakhtenbrot, B., Koss, M. J., et al. 2017, ApJS, 233, 17

Risaliti, G., Nardini, E., Salvati, M., et al. 2011, MNRAS, 410, 1027

Rivers, E., Markowitz, A., Rothschild, R., et al. 2014, ApJ, 786, 126

Sambruna, R. M., Reeves, J. N., Braito, V., et al. 2009, ApJ, 700, 1473

Titarchuk, L. 1994, ApJ, 434, 570

Tortosa, A., Marinucci, A., Matt, G., et al. 2017, MNRAS, 466, 4193

Tortosa, A., Bianchi, S., Marinucci, A., et al. 2018, MNRAS, 473, 3104

Trevese, D., Perna, M., Vagnetti, F., Saturni, F. G., \& Dadina, M. 2014, ApJ, 795 164

Ursini, F., Marinucci, A., Matt, G., et al. 2015, MNRAS, 452, 3266

Veron, P., Lindblad, P. O., Zuiderwijk, E. J., Veron, M. P., \& Adam, G. 1980 A\&A, 87, 245

Vestergaard, M. 2002, ApJ, 571, 733

Vestergaard, M., \& Peterson, B. M. 2006, ApJ, 641, 689

Wang, T., Mihara, T., Otani, C., Matsuoka, M., \& Awaki, H. 1999, ApJ, 515, 567

Wegner, G., Bernardi, M., Willmer, C. N. A., et al. 2003, AJ, 126, 2268

Willmer, C. N. A., Focardi, P., Chan, R., Pellegrini, P. S., \& da Costa, N. L. 1991, AJ, 101, 57

Winter, L. M., Mushotzky, R. F., Reynolds, C. S., \& Tueller, J. 2009, ApJ, 690, 1322

Woo, J.-H., \& Urry, C. M. 2002, ApJ, 579, 530

Zdziarski, A. A., Johnson, W. N., \& Magdziarz, P. 1996, MNRAS, 283, 193

Zhang, E.-P., \& Wang, J.-M. 2006, ApJ, 653, 137

Zoghbi, A., Miller, J. M., Walton, D. J., et al. 2015, ApJ, 799, L24

Życki, P. T., Done, C., \& Smith, D. A. 1999, MNRAS, 309, 561 


\section{Appendix A: Reanalysis of NGC 5506, GRS 1734-292, and MCG-05-23-16}

NGC 5506, GRS 1734-292, and MCG-05-23-16 have the most extreme values of coronal temperature in the sample. These values were obtained from the literature using the COMPTT Comptonization model (Titarchuk 1994). We checked the $k T_{e}$ and $\tau$ values in spherical geometry of the corona for the above sources by reanalysing the NUSTAR observations using the NTHCOMP model (Zdziarski et al. 1996; Życki et al. 1999).

NGC 5506 was observed with NuSTAR (OBSID 60061323) on 2014 April 1. The observation was coordinated with the Swift observatory (OBSID 00080413001), which observed the source on 2012 April 2. In the reanalysis of NGC 5506, we also fitted the simultaneous Swift/XRT data, but we did not re-extract the Swift/XRT spectra.

GRS 1734-292 was observed by NuSTAR on 2014 September 16 (OBSID 60061279002), for a total elapsed time of $43 \mathrm{ks}$.

MCG-05-23-16 was observed on 2012 July 11-12 (OBSID 10002019), and on 2013 June 3-7 (OBSID 60001046). The first observation was conducted as a part of the NuSTAR calibration campaign. The second observation was a science observation carried out simultaneously with a long Suzaku observation. In our reanalysis we only used the NUSTAR science observation.

First, we reduced again the old NuSTAR observations with the NUSTAR Data Analysis Software (NuSTARDAS) package (v. 1.6.0). Cleaned event files (level 2 data products) were then produced and calibrated using standard filtering criteria with the NUPIPELINE task using the last new calibration files available from the NUSTAR calibration database (CALDB 20170120). The extraction radii of the circular region were $0.5 \mathrm{arcmin}$ both for source and for background spectra, for all the sources.

In their analysis of NGC 5506, Matt et al. (2015) found an X-ray spectrum composed of an absorbed power law (with $\Gamma \sim 1.9$ ) with an exponential high-energy cut-off $\left(E_{c}=720_{-190}^{+130} \mathrm{keV}\right)$, plus a moderately ionized reflection component and ionized iron lines. They estimated the coronal parameters using the COMPTT Comptonization model (Titarchuk 1994) and the COMPPS model (Poutanen \& Svensson 1996), and foundin the spherical geometry of the corona-a coronal temperature of $440_{-250}^{+230} \mathrm{keV}(\sim 270 \mathrm{keV})$ and an optical depth of $0.09(0.14)$, respectively.

Tortosa et al. (2017) found, for the NuSTAR spectra of GRS 1734-292, a spectral shape of an absorbed power law with photon index of 1.65 and a very low exponential cut-off, $53_{-8}^{+11} \mathrm{keV}$. They found a reflection fraction of $0.48 \pm 0.22$ and no evidence of relativistic features. Using the COMPTT model and assuming a spherical geometry for the Comptonizing corona, they fond a coronal temperature of $12.1_{-1.3}^{+1.8} \mathrm{keV}$ and an optical depth $\tau=6.38_{-0.5}^{+0.4}$.

The analysis of the NUSTAR spectrum of MCG-05-23-16, performed by Baloković et al. (2015), showed a primary power law with an exponential high-energy cut-off at $116_{-5}^{+6} \mathrm{keV}$, a photon index of $1.85 \pm 0.01$, and the iron line with both narrow and broad components, the last due to relativistic effects. The COMPTT Comptonization model in the case of a spherical corona gives a coronal temperature $k T_{e}=25 \pm 2 \mathrm{keV}$ and a coronal optical depth $\tau=3.5 \pm 0.2$.

We used models similar to those of Matt et al. (2015), Tortosa et al. (2017), and Baloković et al. (2015) to fit the NGC 5506, GRS 1734-292, and MCG-05-23-16 data, but we used the RELXILLCP and XILLVER-COMP models (García \& Kallman 2010; García et al. 2014; Dauser et al. 2014) for the relativistic
Table A.1. List of some parameters obtained from the reanalysis of NGC 5506, GRS 1734-292, and MCG-05-23-16.

\begin{tabular}{ccccc}
\hline \hline Source & $\Gamma$ & $k T_{e}(\mathrm{keV})$ & $\tau$ & $\chi^{2} /$ d.o.f. \\
\hline NGC 5506 & $1.73_{-0.03}^{+0.09}$ & $400 \pm 200$ & 0.21 & 1.1 \\
GRS 1734-292 & $1.81 \pm 0.04$ & $16 \pm 3$ & 3.9 & 1.02 \\
MCG-05-23-16 & $1.93 \pm 0.01$ & $41 \pm 5$ & 1.73 & 1.05 \\
\hline
\end{tabular}

or standard reflection, respectively, with the irradiation of the accretion by a power law with a NTHCOMP (Zdziarski et al. 1996; Życki et al. 1999) Comptonization continuum.

The values obtained with the reanalysis are showed in Table A.1. The coronal optical depth values are extrapolated using the relation from Beloborodov (1999).

The values we found in our reanalysis are different from the literature values, especially the photon index $\Gamma$ (and so the optical depth). However, the error bars on the coronal temperature are almost the same.

Even if the values we found are different from those in the literature, the $\tau-k T_{e}$ pairs follow the relation found previously with the literature values (see Fig. 7).

\section{Appendix B: List of the sources}

- NGC 5506 is a bright, nearby $(z=0.006181)$ Comptonthin (Wang et al. 1999), narrow-line Seyfert 1 galaxy (Nagar et al. 2002). Its spectrum is well described by a power law with $\Gamma=1.9 \pm .03$ with a high-energy exponential cut-off at $720_{-190}^{+130} \mathrm{keV}$ (Matt et al. 2015). NGC 5506 has a galactic absorption with a column density of $3.8 \times 10^{20} \mathrm{~cm}^{-2}$ (Kalberla et al. 2005). The observed 2-10 keV flux corrected for absorption is $6.2 \times 10^{-11} \mathrm{erg} \mathrm{cm}^{-2} \mathrm{~s}^{-1}$ corresponding to a luminosity of $5.26 \times 10^{42} \mathrm{ergs} \mathrm{s}^{-1}$ (Matt et al. 2015).

- MCG-05-23-16 is a nearby $(z=0.0085,36 \mathrm{Mpc})$ Seyfert 1.9 galaxy (Veron et al. 1980; Wegner et al. 2003). This source has a complex structure of the fluorescent line emission, including both broad and narrow components produced by the disc and the torus reflection, respectively (Baloković et al. 2015). It has an absorption with column density of $2.5 \times 10^{22} \mathrm{~cm}^{-2}$. The photon index of the primary power law is found to be $2.00 \pm 0.01$. It also shows a high-energy cut-off at $116_{-5}^{+6} \mathrm{keV}$ (Baloković et al. 2015).

- SWIFT J2127.4+5654 $(z=0.0144)$ is a narrow-line Seyfert 1 galaxy. It was observed by NUSTAR and XMMNewton in an observational campaign performed in November 2012. This source is affected only by the Galactic column density absorption $\left(7.65 \times 10^{21} \mathrm{~cm}^{-2}\right.$, Kalberla et al. 2005). The primary emission of this source has a power-law spectral shape with a photon index of $2.08 \pm 0.01$ and a cut-off energy $E_{c}=108_{-10}^{+11}$ (Marinucci et al. 2014a).

- IC4329A is a nearby bright Seyfert galaxy $(z=0.0161$, Willmer et al. 1991; Galactic $N_{H}=4.61 \times 10^{20} \mathrm{~cm}^{-2}$, Kalberla et al. 2005). It has been observed by NuSTAR almost continuously from 2012 August 12-16. The photon index of the primary power law of IC4329A is $1.73 \pm 0.01$. The spectrum shows a cut-off at $184 \pm 14 \mathrm{keV}$ (Brenneman et al. 2014).

- 3C $390.3(z=0.056)$ is a radio-loud Seyfert 1 galaxy with a weak reflection and a flat photon index. The timing properties of 3C390.3 do not differ from those of radio-quiet 
Seyferts (Gliozzi et al. 2009), and there is no noticeable contribution from the jet to the X-ray emission (Sambruna et al. 2009). It has a Galactic column density of $4 \times 10^{20} \mathrm{~cm}^{-2}$ (Kalberla et al. 2005), a photon index of the primary power law of $1.70 \pm 0.01$, and a cut-off at energy of $116_{-8}^{+24} \mathrm{keV}$ (Lohfink et al. 2015).

- $3 C 382(z=0.057870)$ is a broad-line radio galaxy, but its $\mathrm{X}$-ray continuum is dominated by the Comptonizing corona similarly to radio-quiet Seyfert galaxies (Ballantyne et al. 2014). It has a Galactic absorption with a column density of $N_{H}=6.98 \times 10^{20} \mathrm{~cm}^{-2}$ (Kalberla et al. 2005) and a weak, highly ionized warm absorber with $N_{H} \approx 1.4 \times 10^{21} \mathrm{~cm}^{-2}$ and $\log \xi=.5$; it also has a $\Gamma=1.68_{-0.02}^{+0.03}$ and a high-energy cut-off at $214_{-63}^{+147} \mathrm{keV}$ (Ballantyne et al. 2014).

- GRS 1734-292 $(z=0.0214$, corresponding to a distance of $87 \mathrm{Mpc}$ ) is a Seyfert galaxy originally discovered by the ART-P telescope on board the GRANAT satellite (Pavlinsky et al. 1992). It has a total hydrogen column density in excess of $10^{22} \mathrm{~cm}^{-2}$. The $2-10 \mathrm{keV}$ flux for this source is $F_{2-10}=5.12_{-0.08}^{+0.15} \times 10^{-11} \mathrm{erg} \mathrm{cm}^{-2} \mathrm{~s}^{-1}$. GRS 1734-292 has the spectral slope of the primary power law typical of a Seyfert galaxy in the NUSTAR observation $(\Gamma \sim 1.65)$, with one of the lowest high-energy cut-offs $\left(53_{-8}^{+11} \mathrm{keV}\right)$ measured so far by NuSTAR (Tortosa et al. 2017).

- NGC 6814 ( $z=0.0052$, Molina et al. 2009a) is a Seyfert 1 Galaxy known to show X-ray variability by at least a factor of 10 over timescales of years (Mukai et al. 2003). The 2-10 keV absorption-corrected luminosities from the NuSTAR observation is $\mathrm{L}_{2-10}=2.04 \times 10^{42} \mathrm{erg} \mathrm{s}^{-1}$. It has a primary power law with a photon index of $1.71_{-0.03}^{+0.04}$ and an exponential cut-off at $135_{-35}^{+70} \mathrm{keV}$ (Tortosa et al. 2018).

- MCG 8-11-11 $(z=0.0204)$ is a very X-ray bright AGN. The 2-10 keV absorption-corrected luminosities from the NuSTAR observation is $\mathrm{L}_{2-10}=5.13 \times 10^{43} \mathrm{erg} \mathrm{s}^{-1}$. It has a primary power law with a photon index of $1.77 \pm 0.04$ and an exponential cut-off at $175_{-50}^{+110} \mathrm{keV}$ (Tortosa et al. 2018).

- Ark $564(z=0.02468)$ is a narrow line Seyfert 1 galaxy. It has a steep X-ray spectrum, strong soft excess, and rapid variability. It is also extremely bright in the soft X-ray band $\left(F_{0.3-10 \mathrm{keV}}=1.4 \times 10^{-10} \mathrm{erg} \mathrm{cm}^{-2} \mathrm{~s}^{-1}\right.$ (Kara et al. 2017). Ark 564 has a photon index of $2.27 \pm 0.08$ and a very low cut-off energy value: $E_{c}=42 \pm 3$ (Kara et al. 2017).

- PG 1247+267 is one of the most luminous known quasars at $z \sim 2$ and is a strongly super-Eddington accreting supermassive black hole (SMBH) candidate. It was observed by NUSTAR in December 2014 for a total of 94 ks. From this observation it was found that Pg $1247+267$ has a primary power law with a cut-off energy at $89_{-34}^{+134} \mathrm{keV}$ and photon index of 2.35 $5_{-0.08}^{+0.09}$ (Lanzuisi et al. 2016).

- Ark $120(z=0.033)$ is a "bare" Seyfert 1 galaxy, a system in which ionized, displaying neither intrinsic reddening in its IR continuum nor evidence for absorption in UV and X-rays absorption is absent (Matt et al. 2014; Porquet et al. 2018). The spectrum of the source has a high-energy cut-off value of $183_{-43}^{+83} \mathrm{keV}$ Porquet et al. (2018). The photon index of the primary power law is $1.87 \pm 0.02$ (Porquet et al. 2018).

- NGC $7213(z=0.005839)$ is a low-luminosity AGN that hosts a supermassive black hole of $\sim 10^{8}$ solar masses. It has also been classified as a low-ionization nuclear emission region galaxy (LINER) because of the low excitation observed in the narrow-line spectrum (Filippenko \& Halpern 1984). The photon index of the primary power law of the spectrum of NGC 7213 is $1.84 \pm 0.03$. The source does not have a cut-off measurement, but shows only a lower limit of the cut-off energy of $E_{c}>140 \mathrm{keV}$ (Ursini et al. 2015).

- MCG 6-30-15 $(z=0.008)$, is a Seyfert 1 galaxy with an extreme X-ray variability and a very broad $\mathrm{Fe} \mathrm{k} \alpha$ line emission, with an iron abundance significantly higher than solar (Fabian et al. 2002). Its primary power law shows a photon index of $2.06 \pm 0.01$ and a lower limit on the high energy cut-off that is $>110 \mathrm{keV}$ (Marinucci et al. 2014b).

- NGC $2110(z=0.008)$ is a bright Seyfert 2 galaxy. It shows a prominent $\mathrm{Fe} \mathrm{k} \alpha$ line with a variable intrinsic emission and shows a cut-off energy $E_{c}>210 \mathrm{keV}$ with no detectable contribution from Compton reflection (Marinucci et al. 2015). The source has several layers of absorbing material with column densities in the range $2-6 \times 10^{22} \mathrm{~cm}^{-2}$ (Rivers et al. 2014).

- Mrk $335(z=0.0257)$ is a narrow-line Seyfert 1 galaxy that shows narrower broad emission-line components than a typical Type 1 AGN (Grier et al. 2012). It was observed by NuSTAR in June and July 2013. The Galactic absorption for this source is $3.56 \times 10^{2} \mathrm{~cm}^{-2}$ (Kalberla et al. 2005). Its primary power-law spectrum shows a photon index of $2.14_{-0.04}^{+0.02}$ and a cut-off energy $E_{c}>174$ (Parker et al. 2014).

- Fairall $9(z=0.047016)$ is a Seyfert 1 galaxy. It was observed by NUSTAR in May 2014 and does not show any significant absorption other than Galactic (Lohfink et al. 2016). The photon index of its primary power law is $1.96_{-0.02}^{+0.01}$, which shows a cut-off $E_{c}>242$ (Lohfink et al. 2016).

- Mrk $766(z=0.012929)$ is a narrow line Seyfert 1 galaxy which shows spectral variability in the X-rays (Risaliti et al. 2011). Its X-ray spectrum is well fitted by a power law with photon index $2.22_{-0.03}^{+0.02}$ and an exponential cut-off with a lower limit of $>441 \mathrm{keV}$ (Buisson et al. 2017).

- PG $1211+143(z=0.080900)$ is a bright radio-quiet quasar which belongs to the class of narrow-line Seyfert 1 galaxies and presents an archetypical case for the ultra-fast outflows. The amount of Galactic neutral absorption along the line of sight is $2.7 \times 10^{20} \mathrm{~cm}^{-2}$ (Kalberla et al. 2005). The photon index of the primary power law of the spectrum of this source is $2.51 \pm 0.2$ with a lower limit on the exponential cut-off of $>124 \mathrm{keV}$ (Zoghbi et al. 2015). 